\title{
Quantum master-equation approach to quantum transport through mesoscopic systems
}

\author{
Xin-Qi Li, JunYan Luo, and Yong-Gang Yang \\ State Key Laboratory for Superlattices and Microstructures, Institute of Semiconductors, Chinese Academy of Sciences, P. O. Box 912, \\ Beijing 100083, China \\ Ping Cui and YiJing Yan \\ Department of Chemistry, Hong Kong University of Science and Technology, Kowloon, Hong Kong
}

(Received 27 September 2004; published 2 May 2005)

\begin{abstract}
For quantum transport through mesoscopic systems, a quantum master-equation approach is developed in terms of compact expressions for the transport current and the reduced density matrix of the system. The present work is an extension of Gurvitz's approach for quantum transport and quantum measurement, namely, to finite temperature and arbitrary bias voltage. Our derivation starts from a second-order cumulant expansion of the tunneling Hamiltonian; then follows the conditional average over the electrode reservoir states. As a consequence, in the usual weak-tunneling regime, the established formalism is applicable for a wide range of transport problems. The validity of the formalism and its convenience in application are well illustrated by a number of examples.
\end{abstract}

DOI: 10.1103/PhysRevB.71.205304

PACS number(s): 73.23.-b, 73.63.-b, 72.10.Bg, 72.90.+y

\section{INTRODUCTION}

Quantum transport through mesoscopic nanostructures has revealed many impressive features associated with a number of unique effects such as quantum interferences, discrete levels, and many-body correlations. ${ }^{1}$ Depending on the specific systems or problems under study, theoretical formalisms have been developed such as the Landauer-Büttiker theory and the nonequilibrium Green's function (NGF) approach. ${ }^{1,2}$ However, generally speaking, neither of them implies universal simplicity in practice, for instance, in treating mesosopic transport in the presence of many-electron Coulomb interactions and inelastic scattering with phonons. In particular, it is even more difficult to describe the transient processes (i.e., time-dependent transport phenomena).

In some particular cases, a relatively simpler method that is able to address these issues is the rate-equation approach. ${ }^{3-7}$ Originally, the "classical" rate equation is in a certain sense of phenomenological form. ${ }^{3}$ Later efforts include its derivation and quantum generalization in the context of the resonant tunneling system, based on the NGF quantum kinetic theory, ${ }^{4}$ as well as its modification to describe quantum coherence which typically exists in mesoscopic systems. ${ }^{5,6}$ In particular, a microscopic derivation starting with the many-particle wave function has been presented by Gurvitz and Prager. ${ }^{7}$ However, obvious drawbacks of this approach are its limited validity conditions (i.e., large bias voltage and zero temperature), which greatly restrict the applicability. Also, they were unable to derive a general formula in a "system-Hamiltonian-free" form, which means the inconvenience that one has to proceed with a derivation from the very beginning for every specific system in practice. In this work, we extend Gurvitz's approach to finite temperature and arbitrary bias voltage, as done in our recent work on quantum measurement. ${ }^{8-13}$ In particular, we will establish compact expressions for the transport current together with the reduced density matrix, which can serve as a convenient starting point to study a variety of mesoscopic transport problems.
The remainder of the paper is organized as follows. In Sec. II, starting with the second-order cumulant expansion of the tunneling Hamiltonian, formal expressions for the transport current and the associated master equation are derived. Section III is devoted to a number of examples to illustrate the application of the established formalism. Finally, in Sec. IV concluding remarks on the approximations adopted and the connection with the NGF approach are presented. In the Appendix, a refinement of the cumulant second-order approximation is self-consistently made by including the level broadening effect.

\section{FORMALISM}

Consider the transport setup schematically shown in Fig. 1 which is described by the following Hamiltonian:

$$
\begin{aligned}
H= & H_{S}\left(a_{\mu}^{\dagger}, a_{\mu}\right)+\sum_{\alpha=L, R} \sum_{\mu k} \epsilon_{\alpha \mu k} d_{\alpha \mu k}^{\dagger} d_{\alpha \mu k} \\
& +\sum_{\alpha=L, R} \sum_{\mu k}\left(t_{\alpha \mu k} a_{\mu}^{\dagger} d_{\alpha \mu k}+\text { H.c. }\right) .
\end{aligned}
$$

$H_{S}$ is the (mesoscopic) system Hamiltonian, which can be rather general (e.g., including many-body interaction). $a_{\mu}^{\dagger}$ $\left(a_{\mu}\right)$ is the creation (annihilation) operator of electrons in a state labeled by $\mu$, which labels both the multiorbital and distinct spin states of the system. The second term is the Hamiltonian of the two electrodes, which are also termed the emitter (left electrode) and collector (right electrode) in some parts of this work as usual. The third term describes tunneling between the electrodes and the system. In this paper the electrode reservoir electrons are also given the index $\mu$ to characterize their possible correspondence with the system states. For instance, this will be the typical situation in spindependent transport.

Introducing the reservoir operators $F_{\mu}=\Sigma_{\alpha=L, R} \sum_{k} t_{\alpha \mu k} d_{\alpha \mu k}$ $\equiv f_{L \mu}+f_{R \mu}$, we reexpress the tunneling Hamiltonian as 


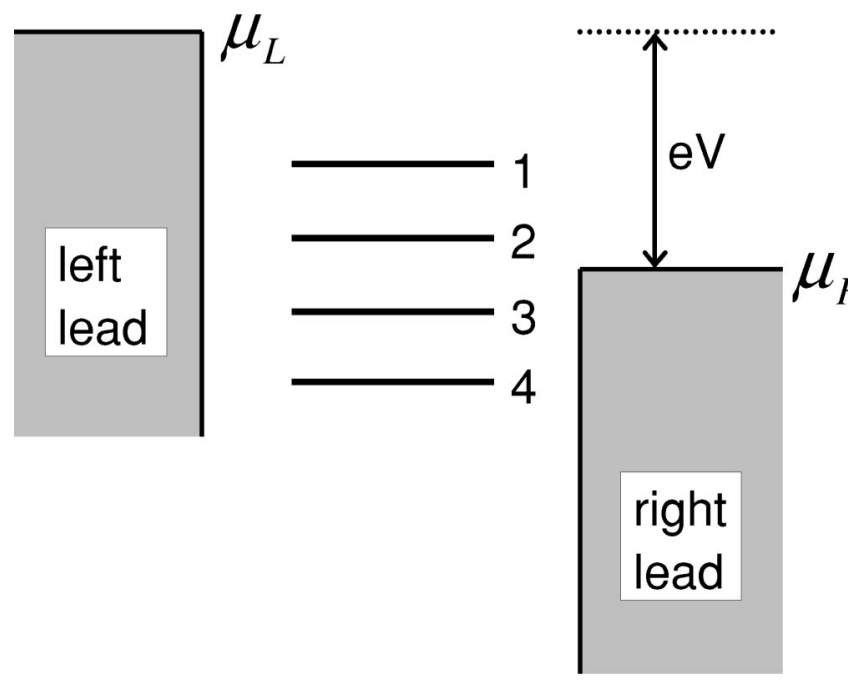

FIG. 1. Schematic setup for electrical transport through a multilevel mesoscopic system.

$$
H^{\prime}=\sum_{\mu}\left(a_{\mu}^{\dagger} F_{\mu}+\text { H.c. }\right)
$$

Then, regarding this tunneling Hamiltonian as a perturbation, the second-order cumulant expansion leads us to a formal equation for the reduced density matrix ${ }^{14}$

$$
\dot{\rho}(t)=-i \mathcal{L} \rho(t)-\int_{0}^{t} d \tau\left\langle\mathcal{L}^{\prime}(t) \mathcal{G}(t, \tau) \mathcal{L}^{\prime}(\tau) \mathcal{G}^{\dagger}(t, \tau)\right\rangle \rho(t) .
$$

Here the Liouvillian superoperators are defined as $\mathcal{L}(\cdots)$ $\equiv\left[H_{S},(\cdots)\right], \quad \mathcal{L}^{\prime}(\cdots) \equiv\left[H^{\prime},(\cdots)\right]$, and $\mathcal{G}(t, \tau)(\cdots) \equiv G(t, \tau)$ $\times(\cdots) G^{\dagger}(t, \tau)$ with $G(t, \tau)$ the usual propagator (Green's function) associated with the system Hamiltonian $H_{S}$. The reduced density matrix $\rho(t)=\operatorname{Tr}_{B}\left[\rho_{T}(\mathrm{t})\right]$, and $\langle(\cdots)\rangle$ $=\operatorname{Tr}_{B}\left[(\cdots) \rho_{B}\right]$ with $\rho_{B}$ the density matrix of the electron reservoirs. Notice that Eq. (3) is nothing but an alternative form of the quantum master equation under the second-order Born approximation. The underlining assumption is that the tunneling Hamiltonian is not strong enough, which makes the second-order cumulant expansion reasonable. It is known that this approximation applies well to most dissipative systems in quantum optics. Noticeably, for most transport systems, weak tunneling is also the typical regime where various forms of golden-rule-type theories are adopted. In the strong-tunneling regime, a special technique is required, which goes beyond the present second-order Born approximation, and other golden-rule-type theories. ${ }^{15}$

The trace in Eq. (3) is over all the electrode degrees of freedom, leading thus to the equation of motion of the unconditional reduced density matrix of the system. To describe the transport problem, we should keep track of the record of electron numbers arriving at the collector (emitted from the emitter and passing through the mesoscopic system in between the two electrodes). We therefore classify the Hilbert space of the electrodes as follows. First, we define the subspace in the absence of electrons arriving at the collector as $B^{(0)}$, which is spanned by the product of all manyparticle states of the two isolated reservoirs, formally denoted as $B^{(0)} \equiv \operatorname{span}\left\{\left|\Psi_{L}\right\rangle \otimes\left|\Psi_{R}\right\rangle\right\}$. Then, we introduce the Hilbert subspace $B^{(n)}(n=1,2, \ldots)$, corresponding to $n$ electrons arriving at the collector. The entire Hilbert space of the two electrodes is $B=\oplus_{n} B^{(n)}$.

With the above classification of the reservoir states, the average over states in the entire Hilbert space $B$ in Eq. (3) is replaced with states in the subspace $B^{(n)}$, leading to a conditional master equation

$$
\begin{aligned}
\dot{\rho}^{(n)}(t)= & -i \mathcal{L} \rho^{(n)}(t)-\int_{0}^{t} d \tau \operatorname{Tr}_{B^{(n)}}\left[\mathcal{L}^{\prime}(t) \mathcal{G}(t, \tau)\right. \\
& \left.\times \mathcal{L}^{\prime}(\tau) \mathcal{G}^{\dagger}(t, \tau) \rho_{T}(t)\right] .
\end{aligned}
$$

Here $\rho^{(n)}(t)=\operatorname{Tr}_{B^{(n)}}\left[\rho_{T}(t)\right]$, which is the reduced density matrix of the system conditioned by the number of electrons arriving at the collector up to time $t$. Now we transform the Liouvillian operator product in Eq. (4) into the conventional Hilbert form:

$$
\begin{aligned}
\mathcal{L}^{\prime}(t) \mathcal{G}(t, \tau) \mathcal{L}^{\prime}(\tau) \mathcal{G}^{\dagger}(t, \tau) \rho_{T}(t)= & {\left[H^{\prime}(t) G(t, \tau) H^{\prime}(\tau) G^{\dagger}(t, \tau) \rho_{T}(t)\right.} \\
& -G(t, \tau) H^{\prime}(\tau) G^{\dagger}(t, \tau) \\
& \left.\times \rho_{T}(t) H^{\prime}(t)\right] \\
& + \text { H.c. } \equiv[I-I I]+\text { H.c. }
\end{aligned}
$$

To proceed, two physical considerations are further implemented as follows. (i) Instead of the conventional Born approximation for the entire density matrix $\rho_{T}(t) \simeq \rho(t) \otimes \rho_{B}$, we propose the ansatz $\rho_{T}(t) \simeq \Sigma_{n} \rho^{(n)}(t) \otimes \rho_{B}^{(n)}$, where $\rho_{B}^{(n)}$ is the density operator of the electron reservoirs associated with $n$ electrons arriving at the collector. With this ansatz for the density operator, tracing over the subspace $B^{(n)}$ yields

$$
\begin{aligned}
\operatorname{Tr}_{B}(n)[I]= & \sum_{\mu, \nu}\left\{\operatorname{Tr}_{B}\left[F_{\mu}^{\dagger}(t) F_{\nu}(\tau) \rho_{B}^{(n)}\right] \times\left[a_{\mu} G(t, \tau) a_{\nu}^{\dagger} G^{\dagger}(t, \tau) \rho^{(n)}\right]\right. \\
& \left.+\operatorname{Tr}_{B}\left[F_{\mu}(t) F_{\nu}^{\dagger}(\tau) \rho_{B}^{(n)}\right] \times\left[a_{\mu}^{\dagger} G(t, \tau) a_{\nu} G^{\dagger}(t, \tau) \rho^{(n)}\right]\right\},
\end{aligned}
$$

$$
\begin{aligned}
\operatorname{Tr}_{B^{(n)}}[I I]= & \sum_{\mu, \nu}\left\{\operatorname{Tr}_{B}\left[f_{L \nu}^{\dagger}(\tau) \rho_{B}^{(n)} f_{L \mu}(t)\right]\right. \\
& \times\left[G(t, \tau) a_{\nu} G^{\dagger}(t, \tau) \rho^{(n)} a_{\mu}^{\dagger}\right]+\operatorname{Tr}_{B}\left[f_{L \nu}(\tau) \rho_{B}^{(n)} f_{L \mu}^{\dagger}(t)\right] \\
& \times\left[G(t, \tau) a_{\nu}^{\dagger} G^{\dagger}(t, \tau) \rho^{(n)} a_{\mu}\right] \\
& +\operatorname{Tr}_{B}\left[f_{R \nu}^{\dagger}(\tau) \rho_{B}^{(n-1)} f_{R \mu}(t)\right] \\
& \times\left[G(t, \tau) a_{\nu} G^{\dagger}(t, \tau) \rho^{(n-1)} a_{\mu}^{\dagger}\right] \\
& +\operatorname{Tr}_{B}\left[f_{R \nu}(\tau) \rho_{B}^{(n+1)} f_{R \mu}^{\dagger}(t)\right] \\
& \left.\times\left[G(t, \tau) a_{\nu}^{\dagger} G^{\dagger}(t, \tau) \rho^{(n+1)} a_{\mu}\right]\right\} .
\end{aligned}
$$

Here we have utilized the orthogonality between states in 
different subspaces, which in fact leads to the term selection from the entire density operator $\rho_{T}$. (ii) Due to the closed nature of the transport circuit, the extra electrons arriving at the collector (right reservoir) will flow back into the emitter (left reservoir) via the external circuit. Also, the rapid relaxation processes in the reservoirs will quickly bring the reservoirs to the local thermal equilibrium state determined by the chemical potentials. As a consequence, after the procedure (i.e., the state selection) done in Eq. (6), the electron reservoir density matrices $\rho_{B}^{(n)}$ and $\rho_{B}^{(n \pm 1)}$ should be replaced by $\rho_{B}^{(0)}$, i.e., the local thermal equilibrium reservoir state, which leads the reservoir correlation functions in Eq. (6) to be, respectively, $\left\langle f_{\alpha \mu}^{\dagger}(t) f_{\alpha \nu}(\tau)\right\rangle=C_{\alpha \mu \nu}^{(+)}(t-\tau)$ and $\left\langle f_{\alpha \mu}(t) f_{\alpha \nu}^{\dagger}(\tau)\right\rangle$ $=C_{\alpha \mu \nu}^{(-)}(t-\tau)$. Here $\langle\cdots\rangle$ stands for $\operatorname{Tr}_{B}\left[(\cdots) \rho_{B}^{(0)}\right]$, with the usual meaning of thermal average. Obviously, $\left\langle F_{\mu}^{\dagger}(t) F_{\nu}(\tau)\right\rangle$ $=C_{\mu \nu}^{(+)}(t-\tau)=\Sigma_{\alpha=L, R} C_{\alpha \mu \nu}^{(+)}(t-\tau)$ and $\left\langle F_{\mu}(t) F_{\nu}^{\dagger}(\tau)\right\rangle=C_{\mu \nu}^{(-)}(t-\tau)$ $=\sum_{\alpha=L, R} C_{\alpha \mu \nu}^{(-)}(t-\tau)$.

Under the Markovian approximation, the time integral in Eq. (4) is replaced by $\frac{1}{2} \int_{-\infty}^{\infty} d \tau$. This approximation considerably simplifies the result. For instance, substituting the first term of Eq. (6) into the time integral of Eq. (4), we have $\int_{-\infty}^{\infty} d \tau C_{\mu \nu}^{(+)}(t-\tau) a_{\mu}\left[e^{-i \mathcal{L}(t-\tau)} a_{\nu}^{\dagger}\right] \rho^{(n)}(t)=a_{\mu}\left[C_{\mu \nu}^{(+)}(-\mathcal{L}) a_{\nu}^{\dagger}\right] \rho^{(n)}(t)$. Other terms can be similarly integrated out, leading to

$$
\begin{aligned}
\dot{\rho}^{(n)}= & -i \mathcal{L} \rho^{(n)}-\frac{1}{2} \sum_{\mu}\left\{\left[a_{\mu}^{\dagger} A_{\mu}^{(-)} \rho^{(n)}+\rho^{(n)} A_{\mu}^{(+)} a_{\mu}^{\dagger}-A_{L \mu}^{(-)} \rho^{(n)} a_{\mu}^{\dagger}\right.\right. \\
& \left.\left.-a_{\mu}^{\dagger} \rho^{(n)} A_{L \mu}^{(+)}-A_{R \mu}^{(-)} \rho^{(n-1)} a_{\mu}^{\dagger}-a_{\mu}^{\dagger} \rho^{(n+1)} A_{R \mu}^{(+)}\right]+ \text {H.c. }\right\} .
\end{aligned}
$$

Here $A_{\alpha \mu}^{( \pm)}=\Sigma_{\nu} C_{\alpha \mu \nu}^{( \pm)}( \pm \mathcal{L}) a_{\nu}$ and $A_{\mu}^{( \pm)}=\Sigma_{\alpha=L, R} A_{\alpha \mu}^{( \pm)}$. The spectral functions $C_{\alpha \mu \nu}^{( \pm)}( \pm \mathcal{L})$ are defined in terms of the Fourier transform of the reservoir correlation functions, i.e., $C_{\alpha \mu \nu}^{( \pm)}( \pm \mathcal{L})$ $=\int_{-\infty}^{\infty} d t C_{\alpha \mu \nu}^{( \pm)}(t) e^{ \pm i \mathcal{L} t}$. We would like to remark here that this time integral leads to "exact" energy conservation for electron transfer between the electrodes and the central system. This "conservation law" would cause errors in the nearresonance bias. For instance, at zero temperature and for electrode Fermi level(s) lower than but very close to a certain system level, the present energy conservation law does not permit any electron occupation on the concerned system level. Nevertheless, the NGF-based quantum kinetic theory allows occupation under the same condition. ${ }^{4}$ The underlying reason is the neglect of level broadening in present treatment, whose inclusion is referred to the Appendix.

The $n$ dependence of Eq. (7) is analogous to the usual rate equation, despite its formal matrix or operator feature. Each term of Eq. (7) can be similarly interpreted as for the conventional " $c$-number" rate equation. Compared with the Bloch equation derived by Gurvitz and Prager, ${ }^{7}$ in Eq. (7) $\rho^{(n)}$ is also coupled to $\rho^{(n+1)}$ which is absent from Ref. 7. This feature originates from the general nature that Eq. (7) is established under nonzero temperature and arbitrary (not necessarily large) bias voltage.

With the knowledge of $\rho^{(n)}(t)$, one is readily able to compute the various transport properties, such as the transport current and noise spectrum. ${ }^{13}$ Remarkably, we can derive a compact expression for the current which is only related to the unconditional density matrix $\rho(t)=\Sigma_{n} \rho^{(n)}(t)$. The deriva- tion is started with the physical observation that the current can be determined by the probability distribution function $P(n, t) \equiv \operatorname{Tr}\left[\rho^{(n)}(t)\right]$, in terms of $I(t)=e d \bar{N}(t) / d t$, where $\bar{N}(t)$ $=\Sigma_{n} n P(n, t)$. Properly treating the summation over $n$ and making use of the cyclic property under the trace, minor algebra based on Eq. (7) straightforwardly leads to

$$
\begin{aligned}
I(t)= & e \sum_{n} n \operatorname{Tr}\left[\dot{\rho}^{(n)}(t)\right] \\
& =\frac{e}{2} \sum_{\mu} \operatorname{Tr}\left[\left(a_{\mu}^{\dagger} A_{R \mu}^{(-)}-A_{R \mu}^{(+)} a_{\mu}^{\dagger}\right) \rho(t)+\text { H.c. }\right] .
\end{aligned}
$$

Here the unconditional density matrix $\rho=\sum_{n} \rho^{(n)}$ satisfies an even simpler equation, which can be easily derived by summing up Eq. (7) over $n$,

$$
\dot{\rho}=-i \mathcal{L} \rho-\frac{1}{2} \sum_{\mu}\left\{\left[a_{\mu}^{\dagger}, A_{\mu}^{(-)} \rho-\rho A_{\mu}^{(+)}\right]+\text {H.c. }\right\} .
$$

Equations (8) and (9) together with Eq. (7) constitute the principal result of this work, which can serve as a convenient starting point to compute the transport current under a wide range of conditions, such as in the presence of many-body Coulomb interactions, at finite temperatures, and for arbitrary voltages. Moreover, the current expression and the associated master equation are free from state representation and the specific system Hamiltonian, which therefore holds the merit of unification in its applications. For instance, for quantum transport through an interacting system, which is usually a challenging problem, one can first diagonalize the isolated system Hamiltonian and then do the Liouvillian operation easily in the eigenstate representation. In the following, as application of this approach we only illustrate a number of simple examples, and retain the systematic applications to more interesting problems to be the subject of forthcoming works.

\section{ILLUSTRATIVE APPLICATIONS}

\section{A. Single-level system}

As a preliminary application of Eq. (7), let us consider the resonant transport through a single-level system. Under the wideband approximation for the electrodes, the reservoir electron correlation functions read $C_{\alpha}^{( \pm)}(t-\tau)$ $=\left|t_{\alpha}\right|^{2} \sum_{k} e^{ \pm i \epsilon_{k}(t-\tau)} n_{\alpha}^{( \pm)}\left(\epsilon_{k}\right)$, where $n_{\alpha}^{(+)}\left(\epsilon_{k}\right)=n_{\alpha}\left(\epsilon_{k}\right)$ is the Fermi distribution function, and $n_{\alpha}^{(-)}\left(\epsilon_{k}\right)=1-n_{\alpha}\left(\epsilon_{k}\right)$. Then the spectral function can be easily carried out as

$$
A_{\alpha}^{( \pm)}=C_{\alpha}^{( \pm)}( \pm \mathcal{L}) a=\Gamma_{\alpha} n_{\alpha}^{( \pm)}\left(E_{0}\right) a .
$$

Here, $\Gamma_{\alpha}=2 \pi g_{\alpha}\left|t_{\alpha}\right|^{2}$, with $g_{\alpha}$ the density of states of the $\alpha$ electrode. In the special case of zero temperature and large bias voltage $\mu_{L} \gg E_{0} \gg \mu_{R}$, which is in fact the applicable condition of Gurvitz and Prager's approach, ${ }^{7}$ we simply have $A_{L}^{(+)}=\Gamma_{L} a, A_{L}^{(-)}=0, A_{R}^{(-)}=\Gamma_{R} a$, and $A_{R}^{(+)}=0$. Substituting these into Eq. (7) yields 


$$
\begin{aligned}
\dot{\rho}^{(n)}= & -i \mathcal{L} \rho^{(n)}-\frac{1}{2}\left\{\left[\Gamma_{R} a^{\dagger} a \rho^{(n)}+\Gamma_{L} \rho^{(n)} a a^{\dagger}-\Gamma_{L} a^{\dagger} \rho^{(n)} a\right.\right. \\
& \left.\left.-\Gamma_{R} a \rho^{(n-1)} a^{\dagger}\right]+ \text { H.c. }\right\} .
\end{aligned}
$$

To obtain the matrix element form of this equation, let us choose the empty (level) state $|0\rangle$ and the occupied state $|1\rangle$ as the representation basis. Straightforwardly, by computing the matrix elements of the terms of Eq. (11) one by one, we obtain

$$
\begin{gathered}
\dot{\rho}_{00}^{(n)}=-\Gamma_{L} \rho_{00}^{(n)}+\Gamma_{R} \rho_{11}^{(n-1)}, \\
\dot{\rho}_{11}^{(n)}=-\Gamma_{R} \rho_{11}^{(n)}+\Gamma_{L} \rho_{00}^{(n)} .
\end{gathered}
$$

This is the result derived by Gurvitz and Prager under the limits mentioned above. ${ }^{7}$

\section{B. Multilevel system}

Now we consider the transport through a multilevel system as shown in Fig. 1, under arbitrary voltage and at finite temperature. The system Hamiltonian simply reads $H_{S}$ $=\sum_{\mu=1}^{N} E_{\mu} a_{\mu}^{\dagger} a_{\mu}$. Also, let us assume that the level separation is much larger than the characteristic level widths, i.e., $\mid E_{\mu}$ $-E_{\mu-1} \mid \gg \Gamma_{L}, \Gamma_{R}$, which leads to the correlation function $C_{\alpha \mu \nu}^{( \pm)}(t) \simeq \delta_{\mu \nu} C_{\alpha \mu \mu}^{( \pm)}(t)$. This assumption neglects the interference effect of electron tunneling through different levels, which is significant only in the case $\left|E_{\mu}-E_{\mu-1}\right|<\Gamma_{L}, \Gamma_{R}$. Similarly to a single-level system, we have $A_{R \mu}^{( \pm)}$ $=\Gamma_{R}\left(E_{\mu}\right) n_{R}^{( \pm)}\left(E_{\mu}\right) a_{\mu}$. For this simplified model, the reduced system density matrix is the direct product of the individual single-level density matrix, i.e., $\rho=\otimes_{\mu=1}^{N} \rho_{\mu}$, and the steadystate solution of the single-level density matrix can be easily obtained as $\rho_{\mu}=p_{\mu}|1\rangle_{\mu}\left\langle 1\left|+\left(1-p_{\mu}\right)\right| 0\right\rangle_{\mu}\langle 0|$, where $|1\rangle_{\mu}\left(|0\rangle_{\mu}\right)$ stands for the occupied (unoccupied) state of the $\mu$ th level, and the occupation probability $p_{\mu}$ reads

$$
p_{\mu}=\frac{n_{L}\left(E_{\mu}\right) \Gamma_{L}\left(E_{\mu}\right)+n_{R}\left(E_{\mu}\right) \Gamma_{R}\left(E_{\mu}\right)}{\Gamma_{L}\left(E_{\mu}\right)+\Gamma_{R}\left(E_{\mu}\right)} .
$$

Substituting the obtained $A_{R \mu}^{( \pm)}$and $\rho$ into the current expression Eq. (8), we arrive at an expression for the steady-state current as

$$
I=e \sum_{\mu} \frac{\Gamma_{L}\left(E_{\mu}\right) \Gamma_{R}\left(E_{\mu}\right)}{\Gamma_{L}\left(E_{\mu}\right)+\Gamma_{R}\left(E_{\mu}\right)}\left[n_{L}\left(E_{\mu}\right)-n_{R}\left(E_{\mu}\right)\right] .
$$

This result clearly manifests the typical steplike $I-V$ characteristics, where each step corresponds to involving a new level in the conduction by increasing the bias voltage, with the standard resonant current $e \Gamma_{L} \Gamma_{R} /\left(\Gamma_{L}+\Gamma_{R}\right)$.

\section{Noninteracting coupled quantum dots}

In the above multilevel system the quantum coherence or nature of quantum superposition of system states is not manifested, and the result can be obtained via classical rate equations. To reveal more clearly the quantum nature of the developed formalism, in this subsection we consider transport through a system of coupled quantum dots. ${ }^{7}$ In this case, the nondiagonal elements of the density matrix, which have no classical counterparts, will appear in the equations of motion and play an essential role.

The Hamiltonian of the coupled quantum dots reads $H_{S}$ $=E_{1} a_{1}^{\dagger} a_{1}+E_{2} a_{2}^{\dagger} a_{2}+\Omega\left(a_{1}^{\dagger} a_{2}+a_{2}^{\dagger} a_{1}\right)$, where each dot contains a single resonant level $E_{1}\left(E_{2}\right)$, and the two dots are coupled by $\Omega$. In principle, for any system the master equation (7) or (9) can be expressed and solved in the system eigenstate representation. Here, for the coupled quantum dots, we would like to present a more elegant method in terms of the language of the Bogoliubov transformation, to explicitly carry out the superoperators $A_{\alpha \mu}^{( \pm)}$. To diagonalize $H_{S}$, the standard Bogoliubov transformation defines a pair of new electron operators as follows: $b_{1}=u a_{1}+v a_{2}$ and $b_{2}=u a_{2}$ $-v a_{1}$. The desired diagonalized Hamiltonian reads $H_{S}$ $=\widetilde{E}_{1} b_{1}^{\dagger} b_{1}+\widetilde{E}_{2} b_{2}^{\dagger} b_{2}$. The diagonalization condition $\left(E_{2}\right.$ $\left.-E_{1}\right) u v+\Omega\left(u^{2}-v^{2}\right)=0$, together with the normalization condition $u^{2}+v^{2}=1$, uniquely determine the transformation coefficients $u$ and $v$, and the eigen-energies read accordingly $\widetilde{E}_{1}=E_{1} u^{2}+E_{2} v^{2}+2 \Omega u v$ and $\widetilde{E}_{2}=E_{1} v^{2}+E_{2} u^{2}-2 \Omega u v$. Simple algebra leads to $\mathcal{L}^{n} a_{1}=\left(-\widetilde{E}_{1}\right)^{n} u b_{1}-\left(-\widetilde{E}_{2}\right)^{n} v b_{2}$ and $\mathcal{L}^{n} a_{2}$ $=\left(-\widetilde{E}_{1}\right)^{n} v b_{1}+\left(-\widetilde{E}_{2}\right)^{n} u b_{2}$. Notice that in the wideband approximation for the electrode reservoirs, $C_{\alpha}^{( \pm)}( \pm \mathcal{L})=\Gamma_{\alpha} n_{\alpha}^{( \pm)}$ $\times(-\mathcal{L})$. We thus have

$$
\begin{aligned}
& A_{L}^{( \pm)}=\Gamma_{L}\left[u n_{L}^{( \pm)}\left(\widetilde{E}_{1}\right) b_{1}-v n_{L}^{( \pm)}\left(\widetilde{E}_{2}\right) b_{2}\right], \\
& A_{R}^{( \pm)}=\Gamma_{R}\left[v n_{R}^{( \pm)}\left(\widetilde{E}_{1}\right) b_{1}+u n_{R}^{( \pm)}\left(\widetilde{E}_{2}\right) b_{2}\right] .
\end{aligned}
$$

With this result, the explicit form of the master equation can be easily obtained for arbitrary offset of the dot levels $\left(E_{1}\right.$ and $E_{2}$ ). To compare with the Bloch equations derived by Gurvitz and Prager, ${ }^{7}$ consider the special configuration of the two dot levels in resonance, i.e., $E_{1}=E_{2} \equiv E_{0}$. For this setup, $u=-v=1 / \sqrt{2}$ and $\widetilde{E}_{1,2}=E_{0} \mp \Omega$. Moreover, in the large-biasvoltage limit $\mu_{L} \gg \widetilde{E}_{2}, \widetilde{E}_{1} \gg \mu_{R}$, we simply have $A_{L}^{(+)}=\Gamma_{L} a_{1}$, $A_{L}^{(-)}=0, A_{R}^{(+)}=0$, and $A_{R}^{(-)}=\Gamma_{R} a_{2}$. Substituting them into Eq. (7), an explicit form of conditional master equation is obtained as

$$
\begin{aligned}
\dot{\rho}^{(n)}= & -i \mathcal{L} \rho^{(n)}-\frac{1}{2}\left\{\left[\rho^{(n)} \Gamma_{L} a_{1} a_{1}^{\dagger}+a_{2}^{\dagger} \Gamma_{R} a_{2} \rho^{(n)}-a_{1}^{\dagger} \rho^{(n)} \Gamma_{L} a_{1}\right.\right. \\
& \left.\left.-\Gamma_{R} a_{2} \rho^{(n-1)} a_{2}^{\dagger}\right]+ \text { H.c. }\right\} .
\end{aligned}
$$

In the electron number representation $\{|1\rangle,|2\rangle,|3\rangle,|4\rangle\}$, which correspond to, respectively, the states of no electron in the two dots, one electron in the left (right) dot, and one electron in each dot, Eq. (16) can be precisely recast to the result derived in Ref. 7, where the quantum coherence nature beyond the classical rate equation was particularly emphasized.

\section{Single-level system in the presence of charging effect}

The above examples do not involve many-electron Coulomb interaction. In this subsection, we consider the simplest example of transport through a single-level system in the presence of the Coulomb charging effect. The system Hamil- 
tonian reads $H_{S}=\Sigma_{\mu}\left[E_{0}+(U / 2) n_{\bar{\mu}}\right] n_{\mu}$. Here the index $\mu$ la-

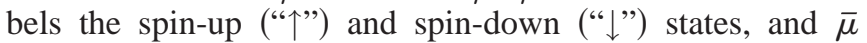
stands for the opposite spin orientation. The electron number operator $n_{\mu}=a_{\mu}^{\dagger} a_{\mu}$ and the Hubbard term $U n_{\uparrow} n_{\downarrow}$ describe the charging effect. Obviously, the reservoir correlation function is diagonal with respect to the spin indices, i.e., $C_{\alpha \mu \nu}^{( \pm)}(t)$ $=\delta_{\mu \nu} C_{\alpha \mu \mu}^{( \pm)}(t)$. We thus have

$$
A_{\alpha \mu}^{( \pm)}=C_{\alpha \mu \mu}^{( \pm)}( \pm \mathcal{L}) a_{\mu}=C_{\alpha \mu \mu}^{( \pm)}\left[\mp\left(E_{0}+U n_{\bar{\mu}}\right)\right] a_{\mu} .
$$

Moreover, for either spin-up or spin-down electrons, the spectral functions $C_{\alpha \mu \mu}^{( \pm)}( \pm E)$ are identical to Eq. (10). For the present system, the four basis states can be chosen as $|1\rangle$ $=|00\rangle_{\uparrow \downarrow},|2\rangle=|10\rangle_{\uparrow \downarrow},|3\rangle=|01\rangle_{\uparrow \downarrow}$, and $|4\rangle=|11\rangle_{\uparrow \downarrow}$. Also in the limiting case of zero temperature and large bias voltage $\left(\mu_{L} \gg E_{0}+U>E_{0} \gg \mu_{R}\right)$, inserting Eq. (17) into Eq. (7) and carrying out the matrix elements associated with the above four basis states, we obtain

$$
\begin{gathered}
\dot{\rho}_{11}^{(n)}=-2 \Gamma_{L} \rho_{11}^{(n)}+\Gamma_{R} \rho_{22}^{(n-1)}+\Gamma_{R} \rho_{33}^{(n-1)}, \\
\dot{\rho}_{22}^{(n)}=-\left(\Gamma_{R}+\Gamma_{L}^{\prime}\right) \rho_{22}^{(n)}+\Gamma_{L} \rho_{11}^{(n)}+\Gamma_{R}^{\prime} \rho_{44}^{(n-1)}, \\
\dot{\rho}_{33}^{(n)}=-\left(\Gamma_{R}+\Gamma_{L}^{\prime}\right) \rho_{33}^{(n)}+\Gamma_{L} \rho_{11}^{(n)}+\Gamma_{R}^{\prime} \rho_{44}^{(n-1)}, \\
\dot{\rho}_{44}^{(n)}=-2 \Gamma_{R}^{\prime} \rho_{44}^{(n)}+\Gamma_{L}^{\prime} \rho_{22}^{(n)}+\Gamma_{L}^{\prime} \rho_{33}^{(n)},
\end{gathered}
$$

where $\Gamma_{\alpha}^{\prime}=\left(2 \pi g_{\alpha}\left|t_{\alpha}\right|^{2}\right)_{E=E_{0}+U}$. Satisfactorily, Eq. (18) is nothing but the result obtained in Ref. 7 under the same limiting conditions.

\section{E. Interacting quantum dots with Zeeman splitting}

In this subsection, we reconsider the model studied in Sec. III D, but slightly modify it by allowing for a finite spin splitting, i.e., $H_{S}=\Sigma_{\mu=\uparrow, ~}\left[E_{\mu} a_{\mu}^{\dagger} a_{\mu}+(U / 2) n_{\mu} n_{\bar{\mu}}\right]$, where the nonzero Zeeman splitting is characterized by $E_{\downarrow}-E_{\uparrow} \equiv \Delta$. The transport properties of this system have been studied recently by Thielmann et al. ${ }^{16}$ by applying the real-time diagrammatic technique. ${ }^{15}$ Here we show that our masterequation approach can solve this nontrivial model in a more transparent way.

As done previously, we first carry out the commutator $\mathcal{L} a_{\mu} \equiv\left[H_{S}, a_{\mu}\right]=-W_{\mu} a_{\mu}, \quad$ where $\quad W_{\mu}=E_{\uparrow} \delta_{\uparrow \mu}+E_{\downarrow} \delta_{\downarrow \mu}$ $+U\left(n_{\uparrow} \delta_{\downarrow \mu}+n_{\downarrow} \delta_{\uparrow \mu}\right)$. Noting that $\left[H_{S}, W_{\mu}\right]=0$, we have $\mathcal{L}^{n} a_{\mu}$ $=\left(-W_{\mu}\right)^{n} a_{\mu}$. Accordingly, $A_{\alpha \mu}^{( \pm)}=C_{\alpha \mu \mu}^{( \pm)}\left(\mp W_{\mu}\right) a_{\mu}$. In the wideband approximation and assuming an energy-independent coupling strength $\Gamma_{L}\left(\Gamma_{R}\right)$ with the left (right) electrode, explicit expressions for $A_{\alpha \mu}^{( \pm)}$are obtained as $A_{L / R \mu}^{( \pm)}=\Gamma_{L / R} n_{L / R}^{( \pm)}$ $\times\left(W_{\mu}\right) a_{\mu}$. In the occupation number representation, i.e., $|1\rangle$ $=|00\rangle_{\uparrow \downarrow},|2\rangle=|10\rangle_{\uparrow \downarrow},|3\rangle=|01\rangle_{\uparrow \downarrow}$, and $|4\rangle=|11\rangle_{\uparrow \downarrow}$, the master equation Eq. (9) can be easily solved, and via Eq. (8) the current can be computed quite straightforwardly. In the following, we explicitly carry out the result in different voltage regimes. For the sake of being able to obtain an analytic result, we focus on the limiting case of zero temperature. Moreover, without loss of generality, we assume that the bias voltage makes the Fermi level of the right electrode always lower than the quantum dot energy levels during transport.
Therefore, all $n_{R}^{(+)}$at the four energies, say, $E_{\uparrow}, E_{\downarrow}, E_{\uparrow}+U$, and $E_{\downarrow}+U$, are zero.

Regime (i). $\mu_{L}>E_{\uparrow}+U, E_{\downarrow}+U, E_{\uparrow}, E_{\downarrow}>\mu_{R}$. In this highbias regime, the corresponding Fermi functions are $n_{L}^{(+)}\left(E_{\uparrow}\right)$ $=n_{L}^{(+)}\left(E_{\downarrow}\right)=n_{L}^{(+)}\left(E_{\uparrow}+U\right)=n_{L}^{(+)}\left(E_{\downarrow}+U\right)=1$, and the master equation Eq. (9) reads

$$
\begin{gathered}
\dot{\rho}_{11}=-2 \Gamma_{L} \rho_{11}+\Gamma_{R} \rho_{22}+\Gamma_{R} \rho_{33}, \\
\dot{\rho}_{22}=-\left(\Gamma_{R}+\Gamma_{L}\right) \rho_{22}+\Gamma_{L} \rho_{11}+\Gamma_{R} \rho_{44}, \\
\dot{\rho}_{33}=-\left(\Gamma_{R}+\Gamma_{L}\right) \rho_{33}+\Gamma_{L} \rho_{11}+\Gamma_{R} \rho_{44}, \\
\dot{\rho}_{44}=-2 \Gamma_{R} \rho_{44}+\Gamma_{L} \rho_{22}+\Gamma_{L} \rho_{33} .
\end{gathered}
$$

To evaluate the stationary current, only a stationary solution is required, which are easily obtained as, respectively, $\rho_{22}$ $=\rho_{33}=\Gamma_{L} \Gamma_{R} /\left(\Gamma_{L}+\Gamma_{R}\right)^{2}, \quad \rho_{11}=\left(\Gamma_{R} / \Gamma_{L}\right) \rho_{22}, \quad$ and $\rho_{44}$ $=\left(\Gamma_{L} / \Gamma_{R}\right) \rho_{22}$. Then, from Eq. (8) the current is simply obtained as

$$
I(t \rightarrow \infty)=e \Gamma_{R}\left(\rho_{22}+\rho_{33}+2 \rho_{44}\right)=\frac{2 e \Gamma_{L} \Gamma_{R}}{\Gamma_{L}+\Gamma_{R}} .
$$

Regime (ii). $E_{\uparrow}+U>\mu_{L}>E_{\downarrow}+U, E_{\uparrow}, E_{\downarrow}>\mu_{R}$. The Fermi functions in this case read $n_{L}^{(+)}\left(E_{\uparrow}+U\right)=0, n_{L}^{(+)}\left(E_{\uparrow}\right)=n_{L}^{(+)}$ $\times\left(E_{\downarrow}\right)=n_{L}^{(+)}\left(E_{\downarrow}+U\right)=1$, and the resulting master equation is

$$
\begin{gathered}
\dot{\rho}_{11}=-2 \Gamma_{L} \rho_{11}+\Gamma_{R} \rho_{22}+\Gamma_{R} \rho_{33}, \\
\dot{\rho}_{22}=-\left(\Gamma_{R}+\Gamma_{L}\right) \rho_{22}+\Gamma_{L} \rho_{11}+\Gamma_{R} \rho_{44}, \\
\dot{\rho}_{33}=-\Gamma_{R} \rho_{33}+\Gamma_{L} \rho_{11}+\Gamma_{R} \rho_{44}+\Gamma_{L} \rho_{44}, \\
\dot{\rho}_{44}=-\left(\Gamma_{L}+2 \Gamma_{R}\right) \rho_{44}+\Gamma_{L} \rho_{22} .
\end{gathered}
$$

Solution of the stationary state reads, respectively, $\rho_{22}$ $=\Gamma_{L} \Gamma_{R}\left(\Gamma_{L}+2 \Gamma_{R}\right) / 2\left(\Gamma_{L}+\Gamma_{R}\right)^{3}, \quad \rho_{33}=\rho_{11}+2 \Gamma_{L}^{2}\left(\Gamma_{L}+\Gamma_{R}\right) / 2\left(\Gamma_{L}\right.$ $\left.+\Gamma_{R}\right)^{3}$, and $\rho_{44}=\Gamma_{L}^{2} \Gamma_{R} / 2\left(\Gamma_{L}+\Gamma_{R}\right)^{3}$. Note that $\rho_{11}=1-\rho_{22}$ $-\rho_{33}-\rho_{44}$, which is irrelevant to the current. Straightforwardly, we obtain the current

$$
I(t \rightarrow \infty)=e \Gamma_{R}\left(\rho_{22}+\rho_{33}+2 \rho_{44}\right)=\frac{e \Gamma_{L} \Gamma_{R}\left(\Gamma_{L}+2 \Gamma_{R}\right)}{\left(\Gamma_{L}+\Gamma_{R}\right)^{2}} .
$$

Regime (iii). $E_{\uparrow}+U, E_{\downarrow}+U>\mu_{L}>E_{\uparrow}, E_{\downarrow}>\mu_{R}$. The Fermi functions $n_{L}^{(+)}\left(E_{\downarrow}\right)=n_{L}^{(+)}\left(E_{\uparrow}\right)=1$ and $n_{L}^{(+)}\left(E_{\uparrow}+U\right)=n_{L}^{(+)}\left(E_{\downarrow}+U\right)$ $=0$. The corresponding master equation reads

$$
\begin{gathered}
\dot{\rho}_{11}=-2 \Gamma_{L} \rho_{11}+\Gamma_{R} \rho_{22}+\Gamma_{R} \rho_{33}, \\
\dot{\rho}_{22}=-\Gamma_{R} \rho_{22}+\Gamma_{L} \rho_{11}+\left(\Gamma_{L}+\Gamma_{R}\right) \rho_{44}, \\
\dot{\rho}_{33}=-\Gamma_{R} \rho_{33}+\Gamma_{L} \rho_{11}+\left(\Gamma_{L}+\Gamma_{R}\right) \rho_{44}, \\
\dot{\rho}_{44}=-2\left(\Gamma_{L}+\Gamma_{R}\right) \rho_{44} .
\end{gathered}
$$

The stationary-state solution of the reduced density matrix leads to the transport current as 


$$
I(t \rightarrow \infty)=e \Gamma_{R}\left(\rho_{22}+\rho_{33}+\rho_{44}\right)=\frac{2 e \Gamma_{L} \Gamma_{R}}{2 \Gamma_{L}+\Gamma_{R}} .
$$

Regime (iv). $E_{\uparrow}+U, E_{\downarrow}+U, E_{\uparrow}>\mu_{L}>E_{\downarrow}>\mu_{R}$. In this setup, only $n_{L}^{(+)}\left(E_{\downarrow}\right)=1$, and all other Fermi functions are zero. Similarly, we first carry out the master equation

$$
\begin{gathered}
\dot{\rho}_{11}=-\Gamma_{L} \rho_{11}+\Gamma_{L} \rho_{22}+\Gamma_{R} \rho_{22}+\Gamma_{R} \rho_{33}, \\
\dot{\rho}_{22}=-\left(\Gamma_{L}+\Gamma_{R}\right) \rho_{22}+\left(\Gamma_{L}+\Gamma_{R}\right) \rho_{44}, \\
\dot{\rho}_{33}=-\Gamma_{R} \rho_{33}+\Gamma_{L} \rho_{11}+\left(\Gamma_{L}+\Gamma_{R}\right) \rho_{44}, \\
\dot{\rho}_{44}=-2\left(\Gamma_{L}+\Gamma_{R}\right) \rho_{44} .
\end{gathered}
$$

Then, the stationary transport current is calculated via the stationary-state solution of the density matrix as

$$
I(t \rightarrow \infty)=e \Gamma_{R}\left(\rho_{22}+\rho_{33}\right)=\frac{e \Gamma_{L} \Gamma_{R}}{\Gamma_{L}+\Gamma_{R}} .
$$

Remarkably, we have precisely recovered all the Coulomb plateaus presented in Ref. 16, which go beyond simple intuition and are obtained there by a not easily accessible realtime diagrammatic technique. This example may shine light on the convenience of our approach in applications.

\section{CONCLUDING REMARKS}

In summary, we have developed an efficient master equation approach for quantum transport through mesoscopic systems, and demonstrated its application by a number of examples. Compared with the previous work by Gurvitz and Prager, ${ }^{7}$ the present study not only generalizes the applicable conditions to finite temperature and arbitrary voltage, but also identifies the adopted approximation which appears not very clear in Ref. 7 . That is, by treating the electrodes as (Fermi) thermal baths, the major approximation adopted in our derivation is the standard second-order Born approximation for the coupling (tunneling) Hamiltonian. It is known that this well-justified approximation makes the resultant quantum master equation applicable in a large number of dissipative systems (e.g., in quantum optics), provided the system-bath coupling is not so strong. Favorably, the illustrated examples in this paper also show its applicability in quantum transport. Moreover, the developed master-equation approach holds the obvious advantages of application convenience and straightforwardness, as well as the ability to address many-body correlation, inelastic scattering, and transient behavior, which are usually difficult issues in mesoscopic transport.

In comparison with the NGF approach, we found that the structure of Eq. (8) is in fact identical to the formal expression of current in terms of nonequilibrium correlation functions. ${ }^{2}$ The NGF approach remains a relatively hard task in searching for particular techniques (e.g., the Feynman diagram or equation of motion) to carry out those correlation functions. In this sense, the obtained Eq. (8) is nothing but the explicit Markovian result under the second-order Born approximation for the tunneling Hamiltonian. In principle, further systematic corrections are possible along the line of going beyond the Born approximation, to include higherorder contributions of tunneling. Finally, we mention that Eq. (8) can be derived from the formal NGF expression of the current; however, the present derivation along the line of Ref. 7 is interesting, and the particular result Eq. (7) from this unique method is of great value, which contains rich information and can be conveniently employed, for instance, to calculate the noise spectrum. ${ }^{9,13,17}$

\section{ACKNOWLEDGMENTS}

Support from the National Natural Science Foundation of China, the Major State Basic Research Project No. G001CB3095 of China, and the Research Grants Council of the Hong Kong Government are gratefully acknowledged.

\section{APPENDIX: LEVEL-BROADENING EFFECT}

To make the derived formulas Eqs. (7)-(9) more accurately applicable to arbitrary voltage, additional care is needed as any of the individual system levels is approaching to the Fermi surface of the electrode. For the sake of description clarity, let us take the single-level resonance system as an example to highlight the key point. As mentioned previously, the treatment in Sec. II under the second-order Born approximation has neglected the level-broadening effect, which would cause certain errors in some particular cases. For instance, current flowing through the resonance system would be strictly forbidden under the near-resonance condition, i.e., as the resonance level $E_{0}$ is a little bit higher than $\mu_{L}\left(\mu_{L}>\mu_{R}\right)$. However, it is well known that a full quantum treatment will give nonzero current in this situation. ${ }^{2,4}$ For a bias voltage such that the resonance level (together with its broadening) is within the range of the two Fermi levels (i.e., $\mu_{L}>E_{0}>\mu_{R}$ ), a common result for the resonance current would be predicted by our master-equation approach and the NGF-based quantum transport theory. In spite of this, it would be desirable to remove the drawback of inaccuracy of our approach in the near-resonance situation.

To account for the level-broadening effect, we return to the evaluation of $A_{\alpha \mu}^{( \pm)}=\Sigma_{\nu} C_{\alpha \mu \nu}^{( \pm)}( \pm \mathcal{L}) a_{\nu}$. Without loss of generality, we restrict our description to the diagonal case $C_{\alpha \mu \nu}^{( \pm)}$ $\times(t)=\delta_{\mu \nu} C_{\alpha \mu \mu}^{( \pm)}(t)$. A more general description is straightforward provided one has clarified the correlation between $f_{\mu}$ and $f_{\nu}$. Using the free-electron-gas model for the electrodes, $A_{\alpha \mu}^{( \pm)}$can be expressed as

$$
A_{\alpha \mu}^{( \pm)}=2 \sum_{k}\left|t_{\alpha \mu, k}\right|^{2} n_{\alpha}^{( \pm)}\left(\epsilon_{k}\right) \int_{0}^{\infty} d t e^{ \pm i \epsilon_{k} t} e^{ \pm i \mathcal{L} t} a_{\mu} .
$$

In our previous treatment, we have replaced the time integral $2 \int_{0}^{\infty} d t$ by $\int_{-\infty}^{\infty} d t$, under the spirit of the Markovian approximation. As a result, the time integration gives rise to a $\delta$ function, $2 \pi \delta\left(\epsilon_{k}+\mathcal{L}\right)$, which characterizes energy conservation for electron transfer between the central system and the electrodes. Mathematically, this procedure is equivalent to dropping the imaginary (principal) part of the integral, and keeping only the real part. Now notice that $e^{ \pm i \mathcal{L} t} a_{\mu}$ describes 
the quantum evolution of the $E_{\mu}$ state (level) associated with the isolated system Hamiltonian. As a standard procedure, the system level-broadening effect due to coupling with the electrodes can be implemented by inserting a damping factor $e^{-\Gamma_{\mu} t}=e^{-\left(\Gamma_{L \mu}+\Gamma_{R \mu}\right) t}$ into the integrand of the time integral. ${ }^{18}$ After this, by keeping only the real part of the integral and adopting the typical wideband approximation for the electrodes, we have

$$
A_{\alpha \mu}^{( \pm)}=\Gamma_{\alpha \mu} \int \frac{d \epsilon_{k}}{2 \pi} \tilde{a}_{\alpha \mu}\left(\epsilon_{k}+\mathcal{L}\right) n_{\alpha}^{( \pm)}\left(\epsilon_{k}\right) a_{\mu}
$$

Here the standard Lorentzian spectral density function reads $\tilde{a}_{\alpha \mu}(\omega+\mathcal{L})=2 \Gamma_{\mu} /\left[(\omega+\mathcal{L})^{2}+\Gamma_{\mu}^{2}\right]$. Formally introducing $N_{\alpha \mu}^{( \pm)}(-\mathcal{L}) \equiv \int d \epsilon_{k}\left(\epsilon_{k} / 2 \pi\right) \tilde{a}_{\alpha \mu}\left(\epsilon_{k}+\mathcal{L}\right) n_{\alpha}^{( \pm)}\left(\epsilon_{k}\right)$, we reexpress (A2) in a very compact form as

$$
A_{\alpha \mu}^{( \pm)}=\Gamma_{\alpha \mu} N_{\alpha \mu}^{( \pm)}(-\mathcal{L}) a_{\mu} .
$$

Elegantly, $N_{\alpha \mu}^{( \pm)}(-\mathcal{L})$ can be regarded as the counterpart of the Fermi function $n_{\alpha}^{( \pm)}(-\mathcal{L})$ after accounting for the level broadening. Combining Eq. (A3) with Eqs. (7)-(9), we complete the generalization of the formalism.

As an illustrative application of the generalized formalism, we reconsider the transport through the (free) multilevel system. Straightforwardly, the current expression of Eq. (14) becomes

$$
\begin{aligned}
I= & e \sum_{\mu} \frac{\Gamma_{L}\left(E_{\mu}\right) \Gamma_{R}\left(E_{\mu}\right)}{\Gamma_{L}\left(E_{\mu}\right)+\Gamma_{R}\left(E_{\mu}\right)} \times \int \frac{d \epsilon_{k}}{2 \pi} \tilde{a}_{\alpha \mu}\left(\epsilon_{k}-E_{\mu}\right) \\
& \times\left[n_{L}\left(\epsilon_{k}\right)-n_{R}\left(\epsilon_{k}\right)\right] .
\end{aligned}
$$

This is the well-known formula for the resonant tunneling current, which is valid for arbitrary voltage including the near-resonance situation.
${ }^{1} \mathrm{~S}$. Datta, Electronic Transport in Mesoscopic Systems (Cambridge University Press, New York, 1995).

${ }^{2}$ H. Haug and A.-P. Jauho, Quantum Kinetics in Transport and Optics of Semiconductors (Springer-Verlag, Berlin, 1996).

${ }^{3}$ L. I. Glazman and K. A. Matveev, JETP Lett. 48, 445 (1988); D. V. Averin and A. N. Korotkov, Sov. Phys. JETP 70, 937 (1990); C. W. J. Beenakker, Phys. Rev. B 44, 1646 (1991).

${ }^{4}$ J. H. Davies, S. Hershfield, P. Hyldgaard, and J. W. Wilkins, Phys. Rev. B 47, 4603 (1993); S. A. Gurvitz, H. J. Lipkin, and Ya. S. Prager, Mod. Phys. Lett. B 8, 1377 (1994).

${ }^{5}$ Yu. V. Nazarov, Physica B 189, 57 (1993).

${ }^{6}$ S. A. Gurvitz, H. J. Lipkin, and Ya. S. Prager, Phys. Lett. A 212, 91 (1996).

${ }^{7}$ S. A. Gurvitz and Ya. S. Prager, Phys. Rev. B 53, 15932 (1996).

${ }^{8}$ S. A. Gurvitz, Phys. Rev. B 56, 15215 (1997).

${ }^{9}$ D. Mozyrsky and I. Martin, Phys. Rev. Lett. 89, 018301 (2002).

${ }^{10}$ S. A. Gurvitz, L. Fedichkin, D. Mozyrsky, and G. P. Berman, Phys. Rev. Lett. 91, 066801 (2003).

${ }^{11}$ R. Ruskov and A. Korotkov, Phys. Rev. B 67, 075303 (2003).
${ }^{12}$ X. Q. Li, W. K. Zhang, P. Cui, J. Shao, Z. Ma, and Y. J. Yan, Phys. Rev. B 69, 085315 (2004).

${ }^{13}$ X. Q. Li, P. Cui, and Y. J. Yan, Phys. Rev. Lett. 94, 066803 (2005).

${ }^{14}$ Y. J. Yan, Phys. Rev. A 58, 2721 (1998).

${ }^{15}$ H. Schoeller and G. Schön, Phys. Rev. B 50, 18436 (1994).

${ }^{16}$ A. Thielmann, M. H. Hettler, J. König, and G. Schön, Phys. Rev. B 68, 115105 (2003).

${ }^{17}$ S. A. Gurvitz, cond-mat/0406010 (unpublished).

${ }^{18} \mathrm{~A}$ more formal and rigorous treatment is possible by improving the second-order Born approximation; namely, by selfconsistently including higher-order contributions of the tunneling Hamiltonian $H^{\prime}$, the operator $\bar{a}_{\mu}(t) \equiv e^{ \pm i \mathcal{L} t-\Gamma_{\mu} t} a_{\mu}$ would satisfy a master-type equation, $\dot{\bar{a}}_{\mu}= \pm i \mathcal{L} \bar{a}_{\mu}-\mathcal{R} \bar{a}_{\mu}$, and the second (damping) term coincides precisely with the one of Eq. (9). This treatment can be termed a self-consistent Born approximation. However, the simple implementation of a damping factor as done in the present Appendix will be more convenient in practice. 